\title{
Altered angiogenesis gene expression in gastrointestinal stromal tumors: potential use in diagnosis, outcome prediction, and treatment
}

\author{
YI ZHAO, Q. WANG*, X. DENG , Y. ZHAO \\ Department of Gastrointestinal Surgery, Shengiing Hospital Affiliated to China Medical University \\ *Correspondence: wangq@sj-hospital.org
}

Received October 21, 2011 / Accepted January 19, 2012

\begin{abstract}
Gastrointestinal stromal tumors (GISTs) occur in diverse locations of the gastrointestinal system. We studied the pathogenesis of GIST by measurement of microvascular density (MVD) and expression of nine signal transduction molecules that have known roles in diverse types of cancers (PI3K, Akt, pTEN, uPA, MMP2, MMP9, HIF1, NOS2, and VEGF) in the tumorous tissues and adjacent normal tissues of 124 GIST patients. We also compared the MVD (a measure of angiogenesis) in tumorous and adjacent normal tissues. Our results indicated significant differences in the expression of the assayed mRNAs and proteins in GIST cells and in adjacent normal cells. In addition, tumorous tissues had significantly higher MVD than adjacent normal tissues. These nine genes have potential for use for diagnosis, outcome prediction, and as new targeted treatments for GISTs.
\end{abstract}

Key words: gastrointestinal stromal tumors (GISTs), angiogenesis, PI3K/AKt signaling pathway, microvascular density (MVD), endothelial growth factor (VEGF), disease-free survival

Gastrointestinal stromal tumors (GISTs) are mesenchymal tumors of the gastrointestinal system, and account for about $2 \%$ of all gastrointestinal malignancies, with an incidence of about 2 per 100000 people [1] There is no strong gender or racial bias, and GISTs can occur anywhere in the gastrointestinal tract, but are most common in the stomach and small intestine and less common in the colorectum and esophagus. GISTs have also been reported in the omentum, mesentery, retroperitoneum, and other parenteral sites [1]. The pathogenesis and biological characteristics of GISTs have not yet been completely described. For non-metastatic GISTs, surgical excision is generally performed [2-3].

Recent studies have implicated $c$-kit in the pathogenesis of GISTs. This gene is a stem cell factor (SFC) receptor that is expressed on the plasma membrane surface; mutated forms of $c$-kit have also been implicated in several different types of cancer [4]. Thus, imatinib (Gleevec), an inhibitor of $c$-kit that was initially approved for treatment of chronic myleogenous leukemia (CML), can also be used to treat GIST [1]. Although imatinib is often effective in the treatment of GIST, most patients eventually develop resistance [5]. This suggests that further characterization of the pathogenesis of GIST may provide a theoretical basis for improved diagnosis, prediction of outcomes, and development of additional targeted therapies.
In the present study, we compared the expression of wellknown tumor-associated signal transduction molecules in GIST tissue and adjacent normal tissue. Although the role of many of these molecules is well-characterized in other systems, their roles in GIST are not yet established. Five of the molecules that we studied have well-known roles in the PI3K/Akt pathway of cancer angiogenesis [6-7]. In this pathway, phosphatidylinositol 3 kinase (PI3K) activates protein kinase B (PKB or Akt), which activates hypoxia-inducible factor- $1 \alpha$ (HIF $1 \alpha)$, which in turn activates vascular endothelial growth factor (VEGF) and urokinase type plasminogen activator (uPA), a serine protease. The four additional molecules that we studied have also well-known roles in cancer pathophysiology in other systems. Phosphatase and tensin homolog (PTEN) is a tumor suppressor gene which, upon mutation, loses function and activates the PI3K/Akt pathway [8]. Matrix-metalloproteinase-2 (MMP2) and MMP9 are involved in extracellular matrix degradation and are believed to have important roles in cancer metastasis [9], and can be activated by uPA [10]. Finally, nitric oxide synthase-2 (NOS2), an inducible isoform of NOS, has diverse roles in signal transduction, including cancer pathogenesis and angiogenesis [11]. Angiogenesis has a well-known role in tumor growth $[12,13]$, so we also compared the microvessel density (MVD) of GIST tissues and adjacent normal tissues. 
Our purpose was to provide important new information about the pathogenesis of GIST, and thereby provide a theoretical basis for improved diagnosis, prediction of outcome, and to guide the development of future targeted therapies.

\section{Patients and Methods}

Patients. We reviewed the records of 124 patients from Shengjing Hospital of China Medical University (Shenyang, Liaoning Province) who had pathologically proven GISTs, complete clinicopathological data, and follow-up data from 1990 to February 2007 (Table 1). There were 64 males and 60 females and the median age was 54.6 years (range: 9-79 years). GISTs were present in the stomach $(n=62,50 \%)$, small intestine $(n=$ $28,22.6 \%)$, colon and rectum $(\mathrm{n}=14,11.3 \%)$, esophagus $(\mathrm{n}=$ $9,7.3 \%$ ), and parenteral sites ( $\mathrm{n}=11,8.9 \%$; 4 in the omentum, 4 in the mesentery, 3 in the retroperitoneum). The criteria used for GIST diagnosis were gross morphology, histopathology, and immunohistochemistry (positive for CD117 (c-kit); CD117, but positive for CD34; or CD117, CD34, SMA;, Desmin, and S-100- for excluding cancers derived from smooth muscle or nerve tissues) [14]. A total of 113 patients were CD117 (91.1\%) and 109 were $\mathrm{CD} 34^{+}(87.9 \%)$. The treatment, including post operative course of chemotherapy, the number of surgical procedures and operation types were also listed in Table1; most patients did not undergo postoperative chemotherapy $(\mathrm{n}=118,95.2 \%)$ and underwent only once surgical procedure $(\mathrm{n}=54,43.5 \%)$. The most frequent operation type was radical resection $(n=95,76.6 \%)$ The median follow-up period was 52 months (range: 10-204 months). Adjacent normal tissues from the same patients were used as controls. All samples were stored at $-80^{\circ} \mathrm{C}$ prior to analysis.

The classification system of Fletcher [14] was used for grading of GISTs. Tumors smaller than $2 \mathrm{~cm}$ in diameter with a mitotic count less than $5 / 50$ were defined as very low risk (Grade I); those that were $2-5 \mathrm{~cm}$ in diameter with a mitotic count less than 5/50 were defined as low risk (Grade II); those that were $5-10 \mathrm{~cm}$ in diameter with a mitotic count less than $5 / 50$ or less than $5 \mathrm{~cm}$ in diameter with a mitotic count of 6 10/50 were defined as intermediate risk (Grade III); and those that were greater than $5 \mathrm{~cm}$ in diameter with a mitotic count
Table 1. Demographic and clinical characteristics of GIST patients $(n=124)$.

\begin{tabular}{ll}
\hline Variable & Number (\%) \\
\hline Gender & \\
Male & 60 \\
Female & 64 \\
Age(mean[range], year) & $54.6(9-79)$ \\
Location & \\
$\quad$ Stomach & $62(50)$ \\
Small Intestine & $28(22.6)$ \\
Colon and rectum & $14(11.3)$ \\
Esophagus & $9(7.3)$ \\
Parental sites & $11(8.9)$ \\
Post operative courses of chemotherapy & \\
0 & $118(95.2)$ \\
2 & $4(3.2)$ \\
4 & $2(1.6)$ \\
Total number of surgical procedures & \\
0 & 0 \\
1 & $54(43.5)$ \\
2 & $28(22.5)$ \\
3 & $26(20.9)$ \\
4 & $10(8.1)$ \\
5 & $2(1.6)$ \\
6 & $1(0.17)$ \\
7 & $3(2.4)$ \\
Imatinib used & $6(4.8)$ \\
Resistance & $1(16)$ \\
Operation type & \\
Unresectable and biopsy & $6(4.8)$ \\
Palliative resection & $23(10.4)$ \\
Rollow up (mean[range], month) & $95(76.6)$ \\
\hline & $52(10-204)$ \\
\hline
\end{tabular}

greater than $5 / 50$ or greater than $10 \mathrm{~cm}$ in diameter with any mitotic count were defined as high risk (Grade IV).

Detection of mRNA expression by RT-PCR. The Illustra ${ }^{\mathrm{TM}}$ QuickPrep mRNA Purification Kit (GE Healthcare, Pittsburgh, USA) was used for RNA extraction and purification. The TaKaRa RNA PCR kit (AMV) ver. 3.0 (Takara, Dalin, China) was used for reverse transcription and amplification according to the manufacturer's instructions. Table 2 lists the PCR

Table 2. Primers used for semi-quantitative RT-PCR analysis.

\begin{tabular}{|c|c|c|c|c|}
\hline Primer & Forward & Reverse & Length (bp) & Cycles \\
\hline PI3K & $5^{\prime}$-accagtaggcaaccgtgaag-3، & $5^{c}$-tggcggatagacatacattgct-3 & 199 & 40 \\
\hline Akt & 5'-gcacaaacgaggggagtacat-3' & $5^{\prime}$-cctcacgttggtccacatc-3' & 113 & 40 \\
\hline PTEN & $5 ’$-tggattcgacttagacttgacct-3' & $5^{\prime}$ - tttggcggtgtcataatgtctt- $3^{\prime}$ & 184 & 40 \\
\hline uPA & 5'-gtgagcgactccaaaggca-3' & 5 '-gcagttgcaccagtgaatgtt- 3 ' & 117 & 40 \\
\hline MMP-2 & $5^{\prime}$ - ccgtcgcccatcatcaagtt-3' & $5^{\prime}$ - ctgtctggggcagtccaaag- $3^{\prime}$ & 169 & 40 \\
\hline MMP-9 & 5'-gggacgcagacatcgtcatc-3' & $5 '$ - tcgtcatcgtcgaaatgggc- $3^{\prime}$ & 139 & 40 \\
\hline NOS2 & 5'-tcatccgctatgctggctac-3' & $5^{\prime}$ - ctcagggtcacggccattg-3' & 161 & 40 \\
\hline VEGF & 5 -caacatcaccatgcagattatgc-3' & $5^{\prime}$-tcggcttgtcacatttttcttgt-3' & 137 & 40 \\
\hline GAPDH & $5^{\prime}$ - atggggaaggtgaaggtcg-3' & 5’-ggggtcattgatggcaacaata-3' & 108 & 40 \\
\hline
\end{tabular}


primers used for the target genes and GAPDH (control). PCR products were separated and visualized by agarose gel electrophoresis, scanned, and quantified by AlphaView gel image analysis software (Santa Clara, USA). The level of GAPDH was measured to confirm equal loading of samples.

Detection of protein expression by western blotting. For detection and quantification of protein, $0.5 \mathrm{~g}$ of GIST tissues were homogenized in $1 \mathrm{~mL}$ of lysis buffer (Cell Signaling Technology, Beverly, MA) with 1 mM PMSF. Proteins were extracted and total protein concentration was determined by the Bradford method. Proteins were mixed with a loading buffer and incubated at $100^{\circ} \mathrm{C}$ for $5 \mathrm{~min}$, separated by $12 \%$ SDS-PAGE, and transferred onto PVDF membranes. These membranes were incubated with primary antibodies and then alkaline phosphatase-conjugated IgG secondary antibodies from ZhongSham GoldenBridge Biotech Ltd. (Beijing). O-dianidine and $\beta$-naphthyl acid phosphate were used as substrates of alkaline phosphatase. Images were scanned quantified by AlphaView gel image analysis software (Santa Clara, USA). All primary antibodies (CD34 [sc-74499], CD117 [sc-3936], PI3K [sc-12929, specific for phosphorylated form], Akt [sc-16646, specific for phosphorylated form], pTEN [sc-73420], uPA [sc-14019], MMP2 [sc-13595], and MMP9 [sc-6841], HIF1 [sc-71247], NOS2 [sc-7271], and VEGF [sc-7269]) were from Santa Cruz Biotechnology Inc. (CA, USA). The level of $\beta$-actin was measured to confirm equal loading of samples.

Measurement of microvascular density. Measurement of microvascular density (MVD) was performed as described by
Weidner [15]. A single brown endothelial cell or cell group was regarded as a blood vessel, but a vessel with a thick muscular layer or with a luminal area greater than the sum of the diameters of 8 red blood cells was not counted as a blood vessel. In each section, the field with the highest MVD was selected at a magnification of 100x, and five fields with CD34-positive vessels were used to determine the average number of vessels. Vascular endothelial cells that were CD34-positive were counted and adjacent normal tissues served as controls.

Immunohistochemical detection of HIF-1a, NOS2 and VEGF. GIST tissues were fixed in formalin, embedded in paraffin, and cut into $4 \mu \mathrm{m}$ sections. Then, deparaffinization was performed, sections were blocked with $50 \mu \mathrm{L}$ of $5 \%$ bovine serum albumin, and incubated with $50 \mu \mathrm{L}$ of primary antibody (see above) and then $50 \mu \mathrm{L}$ of secondary antibody using the Envision $^{\mathrm{TM}}$ FLEX Visualization System (Dako, Glostrup, Denmark). DAB staining for 1-2 min was used for visualization. Under light microscopy, HIF-1a appeared as brown spots in the nucleus and cytoplasm, and NOS2 and VEGF appeared as brown spots in the cytoplasm. CD117 appeared as brown spots on the membrane and CD34 appeared as brown spots in the cytoplasm. Sections were observed by light microscopy at $200 \times$, and hemorrhagic and necrotic areas at the edge of the tumor were excluded. Then, 5 fields with intense staining were selected for cell counting. Scoring of the proportion of positive cells was as follows: 0 , no positive cells; $1,1-25 \%$ positive; 2,26 $50 \%$ positive; $3,51-75 \%$ positive; $4,75-100 \%$ positive. Scoring of staining intensity was as follows: 0 , colorless; 1 , light brown;

Table 3. Expression of mRNAs with known roles in angiogenesis and demographic and clinical characteristics of GIST patients $(n=124)$.

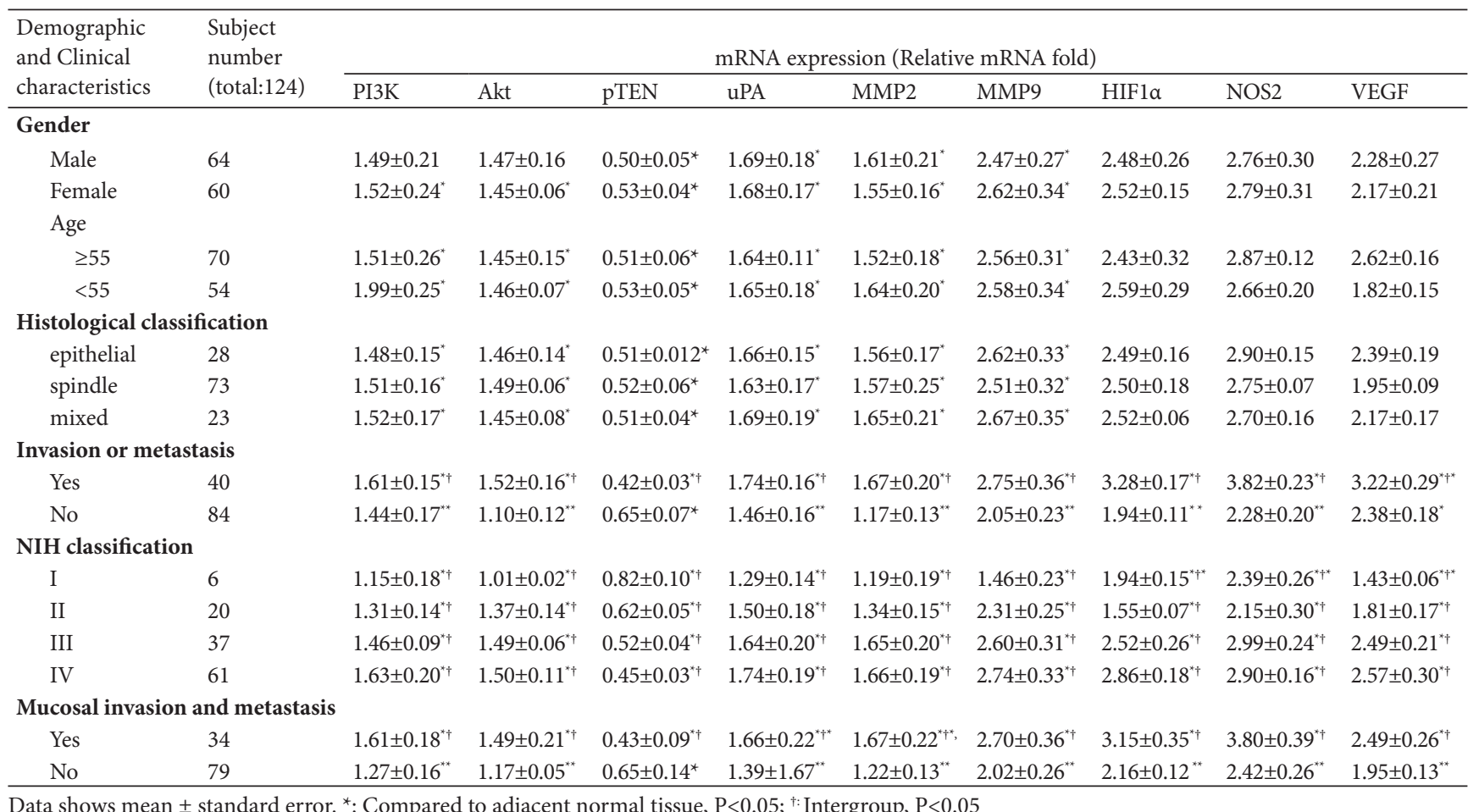

Data shows mean \pm standard error. ${ }^{*}$ : Compared to adjacent normal tissue, $\mathrm{P}<0.05$; ${ }^{\dagger}$ Intergroup, $\mathrm{P}<0.05$ 


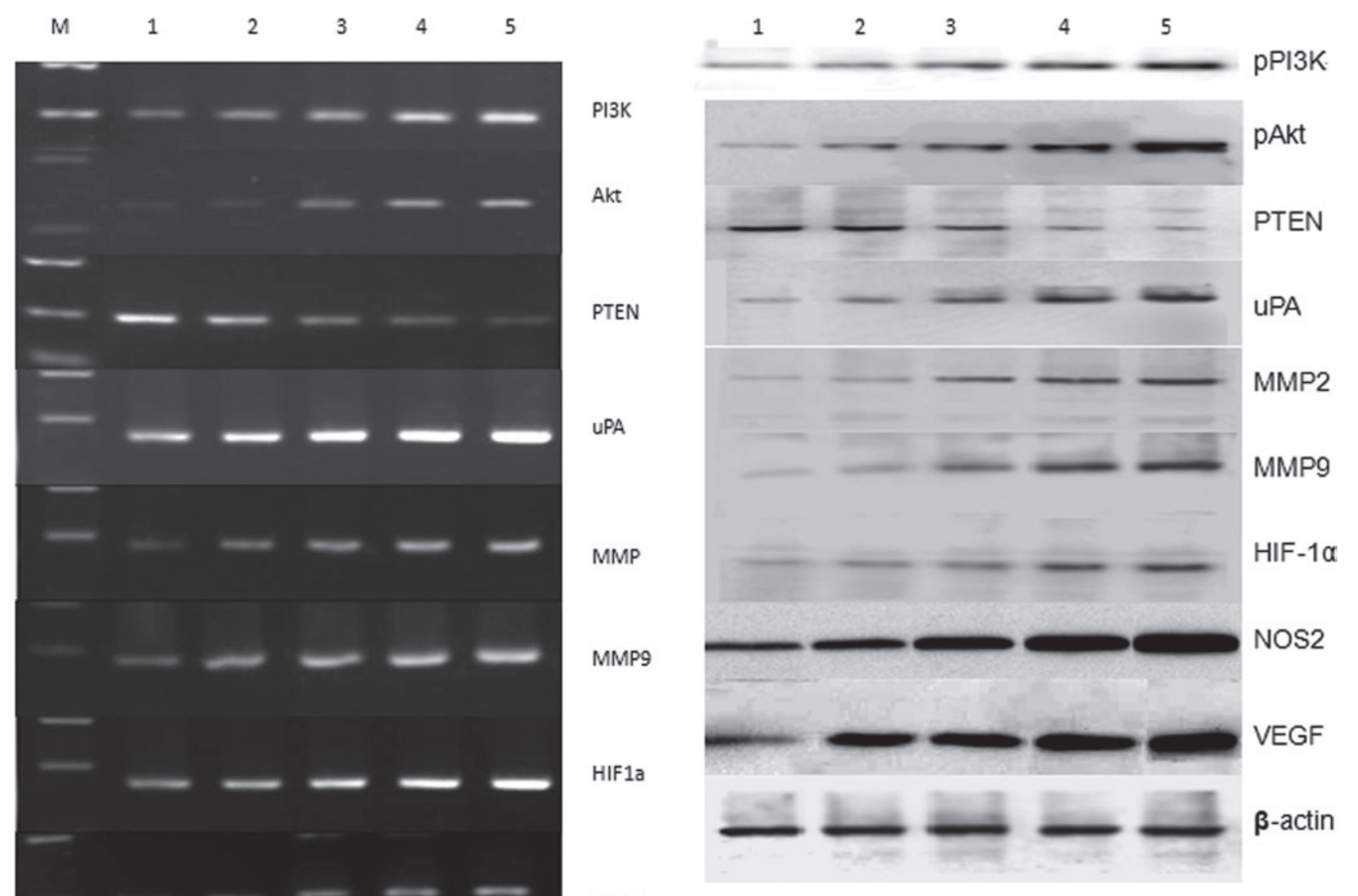

Figure 2. Expression of PI3K, Akt, pTEN, uPA, MMP2, MMP9, HIF1, NOS2, and VEGF proteins in paratumor tissue (1), and in tumors from the very low risk group (2), low risk group (3), middle risk group (4), and high risk group (5) as determined by western blotting.

Figure 1. Expression of PI3K, Akt, pTEN, uPA, MMP2, MMP9, HIF1, NOS2, and VEGF mRNAs in paratumor tissue (1), and in tumors from the very low risk group (2), low risk group (3), middle risk group (4), and high risk group (5) as determined by RT-PCR.

2 , brown; 3 , dark brown. The final score was the product of these two scores: 0 , negative (-); $1-2$, weakly positive $(+) ; 3-4$, positive $(++) ;>4$, strong positive $(+++)$.

Statistics. All experiments were performed 3-4 times, and statistical analysis was conducted with SPSS 13.0 software (Chicago, USA). Survival rate was calculated using the Kaplan-Meier method and the log-rank test was used to compare disease-free survival rates. Multivariate Cox regression analysis was performed for identifying the factors associated with lower disease-free survival rates. A $p$ value less than 0.05 was considered statistically significant.

\section{Results}

Expression of mRNAs. Figure 1 shows representative results of our RT-PCR analysis of the expression of nine genes implicated in cancer pathogenesis in paratumor tissues and tumor tissues with different GIST grades. Table 3 shows the results of our quantitative analysis of this data from 124 GIST patients. For phosphoinositide 3-kinase (PI3K, involved in apoptosis), protein kinase B (Akt, involved in apoptosis), urokinase-type plasminogen activator (uPA, associated with malignancy), matrix metalloproteinase-2 (MMP2, involved in metastasis), and MMP9 (involved in metastasis), mRNA expression was significantly higher in tumorous tissues than in adjacent normal tissues, greater in high grade GISTs than low grade GISTs, and positively associated with the presence of mucosal invasion and metastasis. However, expression of these mRNAs was independent of gender, age, and histological features. These findings suggest that up-regulation of these five genes is associated with the transformation, invasion, and metastasis of GISTs, and therefore implicate the PI3K/Akt signaling pathway in the growth and metastasis of GIST cells. 
In contrast, expression of phosphatase and tensin homologue (pTEN, a tumor suppressor) had a markedly different pattern. PTEN expression was lower in tumorous tissues than in adjacent normal tissues, and was negatively associated with GIST grade and the presence of mucosal invasion and metastasis. Like the other five mRNAs, pTEN expression was independent of age, gender, and histological features.

Expression of proteins. Figure 2 shows representative results of our western blotting analysis of the expression of nine genes implicated in cancer pathogenesis in the paratumor tissue and tumor tissues with different GIST grades. Table 4 shows the results of our quantitative analysis of this data from 124 GIST patients. The results indicate that the expression of hypoxia-inducible factor-1 $\alpha$ (HIF-1 $\alpha$ ), nitric oxide synthase-2 (NOS2), and vascular endothelial growth factor (VEGF), all of which are involved in angiogenesis, were all greater in GIST tissues than adjacent normal tissues, and that all were positively associated with tumor grade, mucosal invasion, and metastasis. However, expression of these proteins was not significantly related to patient age, gender, or other clinicopathological features (Table 4).

HIF-1a expression was positive in $46.2 \%$ of patients with grade I/II GIST and in $68.4 \%$ of patients with grade III/IV GIST ( $p=0.006)$; in $54.4 \%$ of patients without mucosal invasion and $88.2 \%$ of patients with mucosal invasion $(p=0.017)$; and in $51.2 \%$ of patients without metastasis and $90.0 \%$ of patients with metastasis $(p=0.026)$.
VEGF expression was elevated in $46.2 \%$ of patients with grade I/II GIST and $61.2 \%$ of patients with grade III/IV GIST ( $p=0.008)$; in $50.6 \%$ of patients without mucosal invasion and $79.4 \%$ of patients with mucosal invasion $(p=0.006)$; and in $47.6 \%$ of patients without metastasis and $80.0 \%$ of patients with metastasis $(p=0.015)$.

NOS2 expression was elevated in $42.3 \%$ of patients with grade I/II GIST and $70.4 \%$ in patients with grade III/IV GIST $(p=0.039)$; in $55.7 \%$ of patients without mucosal invasion and $88.2 \%$ in patients with mucosal invasion $(p=0.003)$; and in $50.0 \%$ in patients without metastasis and $95.0 \%$ of patients with metastasis $(p=0.042)$.

Taken together, these immunohistochemical findings suggest that HIF-1 $\alpha$, NOS2, and VEGF are all involved in the occurrence and development of GISTs, and therefore have an important role in the angiogenesis, growth, and metastasis of GIST.

Microvascular density. Figure 4 is a representative image showing the MVD of a GIST. Table 5 shows that among our 124 GIST patients, there was a significant positive association of MVD and the presence of GIST. Moreover, the MVD was significantly greater in high grade tumors and greater in tumors that had mucosal invasion or metastasis. There were no significant effects of gender, age, or histopathological classification. Thus, in agreement with our semi-quantitative RT-PCR results and western blotting results (Table 4), these results indicate that angiogenesis is more active in GIST tissues

Table 4. Expression of proteins with known roles in angiogenesis and demographic and clinical characteristics of GIST patients $(\mathrm{n}=124)$.

\begin{tabular}{|c|c|c|c|c|c|c|c|c|c|c|}
\hline \multirow{2}{*}{$\begin{array}{l}\text { Demographic and Clini- } \\
\text { cal characteristics }\end{array}$} & \multirow{2}{*}{$\begin{array}{l}\text { Subject } \\
\text { number } \\
\text { (total:124) }\end{array}$} & \multicolumn{9}{|c|}{ Protein expression (percent increase relative to adjacent normal tissue) } \\
\hline & & $\begin{array}{l}\text { PI3K, } \\
\text { phosporylated }\end{array}$ & $\begin{array}{l}\text { Akt, } \\
\text { phosphorylated }\end{array}$ & pTEN & $\mathrm{uPA}$ & MMP2 & MMP9 & HIF1a & NOS2 & VEGF \\
\hline \multicolumn{11}{|l|}{ Gender } \\
\hline Male & 64 & $72 \pm 13$ & $90 \pm 19$ & $75 \pm 10^{*}$ & $60 \pm 10^{*}$ & $45 \pm 13^{*}$ & $75 \pm 12^{*}$ & $54 \pm 11$ & $81 \pm 14$ & $96 \pm 18$ \\
\hline Female & 60 & $75 \pm 11^{*}$ & $105 \pm 20^{*}$ & $80 \pm 14^{*}$ & $65 \pm 15^{*}$ & $58 \pm 10^{*}$ & $78 \pm 15^{*}$ & $65 \pm 12$ & $95 \pm 10$ & $100 \pm 14$ \\
\hline \multicolumn{11}{|l|}{ Age } \\
\hline$\geq 55$ & 70 & $70 \pm 10^{*}$ & $95 \pm 18^{*}$ & $85 \pm 16^{*}$ & $70 \pm 11^{*}$ & $55 \pm 12^{*}$ & $56 \pm 13^{*}$ & $72 \pm 15$ & $78 \pm 5$ & $92 \pm 15$ \\
\hline$<55$ & 54 & $65 \pm 12^{*}$ & $100 \pm 19^{*}$ & $75 \pm 10^{*}$ & $60 \pm 15^{*}$ & $60 \pm 14^{*}$ & $65 \pm 15^{*}$ & $80 \pm 12$ & $85 \pm 16$ & $85 \pm 11$ \\
\hline \multicolumn{11}{|l|}{$\begin{array}{l}\text { Histological classifica- } \\
\text { tion }\end{array}$} \\
\hline epithelial & 28 & $75 \pm 11^{*}$ & $90 \pm 17^{*}$ & $78 \pm 5^{*}$ & $75 \pm 10^{*}$ & $50 \pm 11^{*}$ & $80 \pm 14^{*}$ & $55 \pm 10$ & $69 \pm 11$ & $107 \pm 12$ \\
\hline spindle & 73 & $80 \pm 14^{*}$ & $85 \pm 16^{*}$ & $92 \pm 15^{*}$ & $80 \pm 14^{*}$ & $65 \pm 15^{*}$ & $75 \pm 11^{*}$ & $60 \pm 9$ & $75 \pm 10$ & $115 \pm 21$ \\
\hline mixed & 23 & $70 \pm 12^{*}$ & $95 \pm 17^{*}$ & $105 \pm 15^{*}$ & $65 \pm 16^{*}$ & $70 \pm 10^{*}$ & $66 \pm 12^{*}$ & $64 \pm 15$ & $64 \pm 11$ & $95 \pm 15$ \\
\hline \multicolumn{11}{|l|}{ Invasion or metastasis } \\
\hline Yes & 40 & $120 \pm 19^{* \dagger}$ & $150 \pm 30^{* \dagger}$ & $75 \pm 11^{* \dagger}$ & $110 \pm 20^{* \dagger}$ & $120 \pm 20^{* \dagger}$ & $135 \pm 25^{* \dagger}$ & $105 \pm 10^{* \dagger}$ & $110 \pm 15^{* \dagger}$ & $165 \pm 34^{*+*}$ \\
\hline No & 84 & $90 \pm 17^{* *}$ & $100 \pm 20^{* *}$ & $120 \pm 10^{*}$ & $50 \pm 15^{* *}$ & $55 \pm 15^{* *}$ & $45 \pm 15^{* *}$ & $71 \pm 5^{* *}$ & $78 \pm 16^{* *}$ & $110 \pm 25^{*}$ \\
\hline \multicolumn{11}{|l|}{ NIH classification } \\
\hline I & 6 & $52 \pm 16^{* \dagger}$ & $70 \pm 10^{*+}$ & $100 \pm 10^{* \dagger}$ & $45 \pm 13^{* \dagger}$ & $35 \pm 10^{*+}$ & $36 \pm 11^{* \dagger}$ & $37 \pm 10^{*+*}$ & $48 \pm 10^{*+*}$ & $85 \pm 16^{* *^{*}}$ \\
\hline II & 20 & $80 \pm 17^{\star \dagger}$ & $90 \pm 15^{*}$ & $80 \pm 11^{*+}$ & $88 \pm 17^{* \dagger}$ & $68 \pm 15^{*+}$ & $85 \pm 16^{* \dagger}$ & $46 \pm 11^{* \dagger}$ & $50 \pm 12^{* \dagger}$ & $110 \pm 21^{* \dagger}$ \\
\hline III & 37 & $95 \pm 19^{*+}$ & $119 \pm 18^{* \dagger}$ & $60 \pm 7^{* \dagger}$ & $115 \pm 24^{* \dagger}$ & $101 \pm 20^{* \dagger}$ & $125 \pm 26^{* \dagger}$ & $85 \pm 12^{* \dagger}$ & $84 \pm 11^{* \dagger}$ & $121 \pm 10^{* \dagger}$ \\
\hline IV & 61 & $120 \pm 22^{* \dagger}$ & $125 \pm 21^{* \dagger}$ & $45 \pm 11^{*+}$ & $122 \pm 20^{* \dagger}$ & $115 \pm 20^{* \dagger}$ & $140 \pm 25^{* \dagger}$ & $94 \pm 19^{* \dagger}$ & $105 \pm 18^{* \dagger}$ & $142 \pm 22^{* \dagger}$ \\
\hline \multicolumn{11}{|c|}{ Mucosal invasion and metastasis } \\
\hline Yes & 34 & $115 \pm 21^{* \dagger}$ & $130 \pm 15^{* \dagger}$ & $65 \pm 10^{*+}$ & $120 \pm 18^{* \dagger}$ & $110 \pm 16^{*}$ & $130 \pm 16^{* \dagger}$ & $110 \pm 15^{* \dagger}$ & $120 \pm 11^{* \dagger}$ & $155 \pm 24^{* \dagger}$ \\
\hline No & 79 & $70 \pm 14^{* *}$ & $105 \pm 15^{* *}$ & $110 \pm 20^{*}$ & $65 \pm 10^{* *}$ & $55 \pm 12^{* *}$ & $72 \pm 13^{* *}$ & $60 \pm 11^{* *}$ & $65 \pm 10^{* *}$ & $70 \pm 16^{* *}$ \\
\hline
\end{tabular}

Data shows mean \pm standard error. ${ }^{*}$ : Compared to adjacent normal tissue, $\mathrm{P}<0.05$; ${ }^{\dagger}$ Intergroup, $\mathrm{P}<0.05$ 

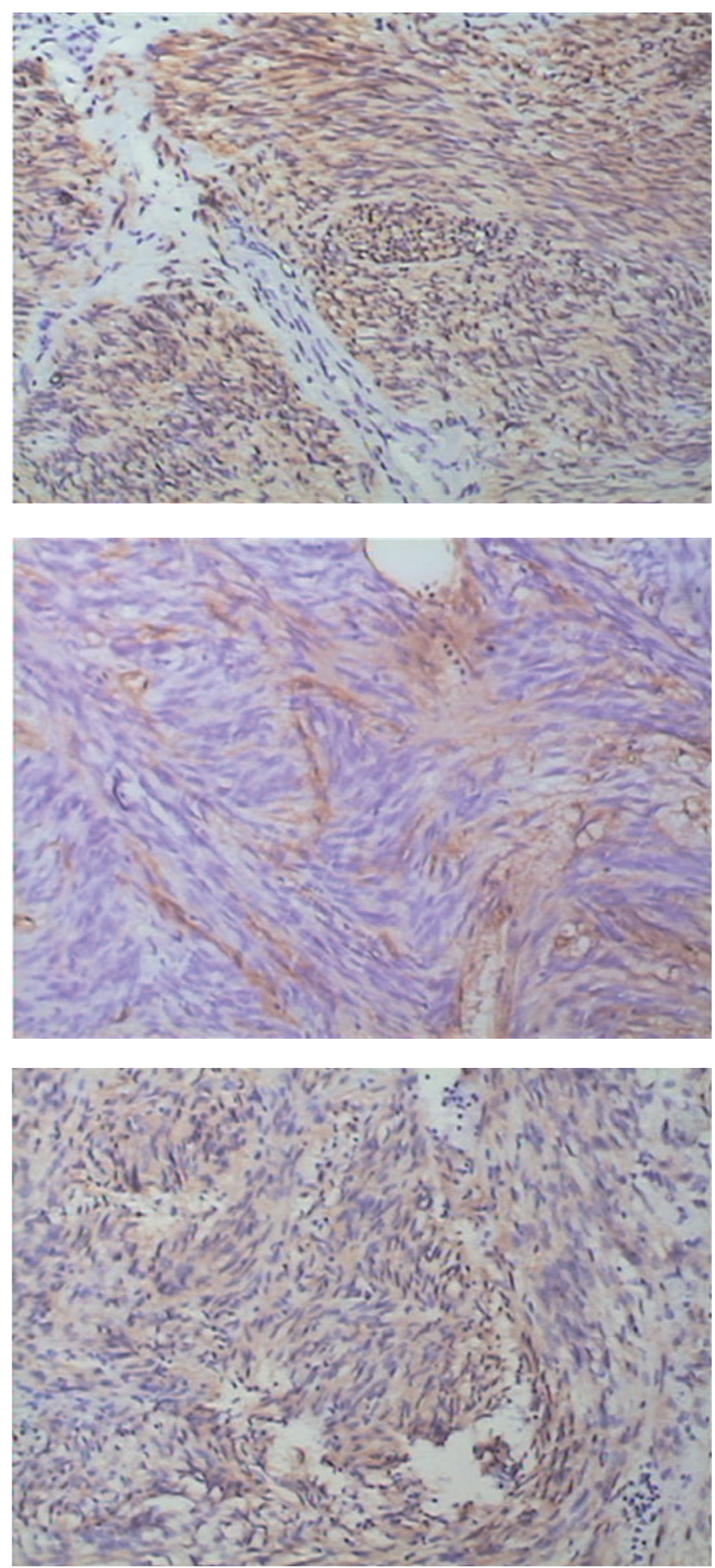

Figure 3. Expression of HIF-1a (A), NOS2 (B), and VEGF (C) in representative GISTs, as determined by immunohistochemistry $(\times 200)$.

than in adjacent normal tissues. As expected, the disease-free survival of patients was poorer when there was extensive MVD, tumor metastasis, mucosal invasion, and higher grade tumors. Correlation analysis also indicated that VEGF and HIF1 $\alpha$ were

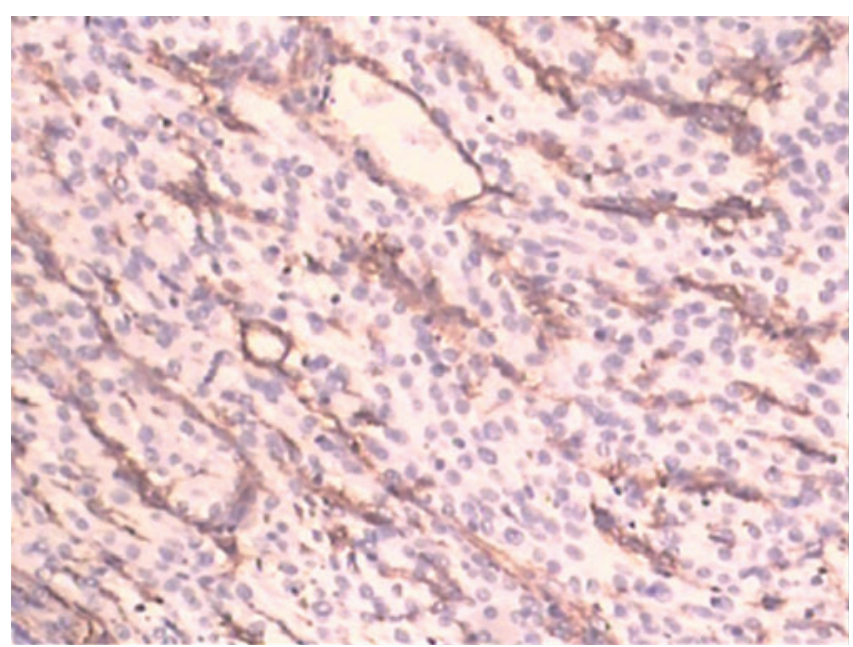

Figure 4. Representative micro-vessel density, determined by immunohistochemical analysis of CD34 $(\times 200)$.

positively correlated with MVD $(\mathrm{r}=0.324, p=0.021$ and $\mathrm{r}=$ $0.412, p=0.034$, respectively), but NOS2 was not significantly correlated with MVD.

Finally, we performed a multivariate analysis based on the protein expression results to identify factors independently associated with disease free survival. Our results indicate that NIH classification, MVD value, and expression of PI3K, PTEN, MMP9, HIF1 $\alpha$, and VEGF were independently associated with lower disease-free survival (Table 6, Supplementary Figure 1).

\section{Discussion}

The prognoses of patients with GISTs are affected by several factors, most importantly by tumor grade and the use of targeted therapy [16]. Sustained activation of the PI3K/AKt signaling pathway is known to be related to the occurrence and development of GIST [17], and mutation of PTEN is known to have a role in activation of this pathway [18]. However, little is known to date about the role of the PI3K/AKt pathway and PTEN in the prognosis of patients with GIST. Our results suggest that up-regulation of the PI3K/AKt pathway may be a predictor of GIST and that measurement of known signal transduction factors involved in numerous other cancers may provide important information on the prognosis of patients with GISTs.

Cancer angiogenesis is an important prerequisite for metastasis of GISTs and other tumors, and the PI3K/AKt signaling pathway is involved in this process. For example, HIF-1a binds to HIF-response elements in the promoters of numerous genes, including VEGF, a protein with well-known roles in cancer angiogenesis and metastasis [19]. The PI3K/ AKt signaling pathway can regulate HIF-1a expression via PTEN; inactivation of PTEN may activate AKt which then stabilizes the HIF-1a [20] and promotes the transcription of 
Table 5. Microvessel density of GIST patients $(n=124)$.

\begin{tabular}{|c|c|c|c|}
\hline Demographic/clinical characteristic & Subject number (total:124) & MVD & Disease free survival(DFS) \\
\hline \multicolumn{4}{|l|}{ Gender } \\
\hline Male & 64 & $24.6 \pm 8.56$ & $40.81 \pm 15.66$ \\
\hline Female & 60 & $21.7 \pm 6.24$ & $38.62 \pm 16.75$ \\
\hline \multicolumn{4}{|l|}{ Age } \\
\hline$\geq 55$ & 70 & $22.5 \pm 9.53$ & $36.73 \pm 13.22$ \\
\hline$<55$ & 54 & $23.8 \pm 8.69$ & $39.51 \pm 14.10$ \\
\hline \multicolumn{4}{|l|}{ Histological classification } \\
\hline epithelial & 28 & $21.3 \pm 6.92$ & $37.65 \pm 9.66$ \\
\hline spindle & 73 & $26.8 \pm 10.01$ & $39.87 \pm 10.50$ \\
\hline mixed & 23 & $25.7 \pm 11.32$ & $35.74 \pm 11.32$ \\
\hline \multicolumn{4}{|l|}{ Invasion or metastasis } \\
\hline Yes & 40 & $30.35 \pm 6.98^{*+}$ & $30.80 \pm 9.59^{* \dagger}$ \\
\hline No & 84 & $23.22 \pm 6.07^{*}$ & $46.08 \pm 19.75^{*}$ \\
\hline \multicolumn{4}{|l|}{ NIH classification } \\
\hline I & 6 & $14.7 \pm 4.65^{* \dagger}$ & $70.50 \pm 15.66^{* \dagger}$ \\
\hline II & 20 & $18.59 \pm 6.89^{* \dagger}$ & $54.95 \pm 17.89^{* \dagger}$ \\
\hline III & 37 & $22.76 \pm 7.21^{* \dagger}$ & $48.11 \pm 15.28^{* \dagger}$ \\
\hline IV & 61 & $30.64 \pm 7.72^{* \dagger}$ & $29.28 \pm 10.80^{* \dagger}$ \\
\hline \multicolumn{4}{|l|}{ Mucosal invasion and metastasis } \\
\hline Yes & 34 & $30.85 \pm 7.66^{* \dagger}$ & $30.33 \pm 9.38^{*+}$ \\
\hline No & 79 & $22.58 \pm 8.44^{*}$ & $47.13 \pm 19.65^{*}$ \\
\hline Adjacent normal tissues & 124 & $8.3 \pm 2.76$ & - \\
\hline
\end{tabular}

Data shows mean \pm standard error. ${ }^{*}$ : Compared to adjacent normal tissue, $\mathrm{P}<0.05$; ${ }^{\star}$ : Intergroup, $\mathrm{P}<0.05$

Table 6. Multivariate analysis of factors associated with disease-free survival.

\begin{tabular}{llll}
\hline Factors & P value & HR & $95 \%$ CI(range) \\
\hline Gender & 0.519 & 0.992 & $0.967-1.017$ \\
Age & 0.701 & 1.565 & $1.104-2.767$ \\
Histological classification & 0.113 & 3.461 & $0.807-10.504$ \\
Invasion or metastasis & 0.198 & 0.503 & $0.176-1.433$ \\
NIH classification & 0.007 & 4.624 & $1.822-9.778$ \\
Mucosal invasion & 0.143 & 3.626 & $0.906-6.751$ \\
Expression of PI3K & 0.04 & 3.565 & $1.956-28.794$ \\
Expression of Akt & 0.03 & 3.987 & $0.906-6.545$ \\
Expression of PTEN & 0.001 & 6.591 & $1.265-29.694$ \\
Expression of uPA & 0.225 & 1.346 & $0.867-1.216$ \\
Expression of MMP2 & 0.429 & 0.324 & $0.505-2.361$ \\
Expression of MMP9 & 0.749 & 0.935 & $1.304-3.776$ \\
Expression of HIF1a & 0.03 & 5.435 & $1.956-32.678$ \\
Expression of NOS2 & 0.213 & 2.346 & $1.766-3.768$ \\
Expression of VEGF & 0.002 & 8.561 & $1.132-23.465$ \\
MVD value & $<0.001$ & 9.605 & $3.107-11.185$ \\
\hline
\end{tabular}

hypoxia-responsive genes. This in turn elicits a series of adaptive responses to hypoxia, including angiogenesis, increased vascular permeability, and then metastasis [21].

GISTs have rich blood supplies and hematogenous dissemination, and metastasis is closely related to VEGF expression [22]. In the present study, we showed that VEGF expression was strongly associated with tumor grade, mucosal invasion, and metastasis. These findings strongly suggest that VEGF plays a critical role in the pathogenesis of GISTs. VEGF expression is known to be closely related to MVD. These findings indicated that VEGF is involved in the occurrence, development and metastasis of GIST through regulation of angiogenesis [23]. Because VEGF is closely related to the growth, metastasis, and prognosis of GISTs, we suggest that it be considered as a target for the treatment of this cancer. Suppression of VEGF expression may inhibit cancer angiogenesis, and decrease blood supply to cancers and metastasis leading to improved prognosis. Therapies targeting VEGF have been considered for treatment of neuroblastoma [24].

Evidence has shown that NOS activity, especially NOS2 activity, is markedly increased in numerous solid cancers and cancer cell lines. For example, NOS expression is up-regulated in prostate cancer, breast cancer, bladder cancer, and gynecological cancers and NOS has been proposed as a therapeutic target for treatment of cancer [25]. In the present study, we found that NOS2 expression was positively associated with GIST grade, mucosal invasion, and metastasis $(p<0.05)$. Our findings also indicated that HIF- $1 \alpha$ and NOS2 expression were positively correlated with VEGF expression $(\mathrm{r}=0.368, p<$ $0.05, r=0.452, p<0.05$, respectively). Therefore, we suggest that up-regulation of VEGF increases the invasion, metastasis and angiogenesis of GISTs. Thus, HIF-1 $\alpha$, NOS2, and VEGF might be important indicators of GIST growth, invasion, and metastasis and may also be potential therapeutic targets. 
The interactions of all of these factors may be summarized as: (i) HIF-1a up-regulates downstream genes (such as VEGF [61]) and promotes angiogenesis and vascular permeability, allowing cancer invasion; (ii) VEGF (but not bFGF) regulates angiogenesis through the NO/cGMP signaling pathway; (iii) VEGF may induce NO production by activating the PI3K/AKt signaling pathway. Our study showed that NOS2 expression was positively correlated with VEGF expression $(\mathrm{r}=0.452$, $p=0.021)$. NO can activate intracellular guanylate cyclase and increase the level of cGMP, the main effector molecule of $\mathrm{NO}$, and can thereby stimulate angiogenesis. Although these factors can regulate angiogenesis, few studies have examined the mechanisms underlying the angiogenesis of GISTs.

Clearly, angiogenesis plays a critical role in the invasion and metastasis of GISTs, and expression of factors related to angiogenesis are closely related to the degree of malignancy. In the present study, we showed that HIF-1 $\alpha$, NOS2, and VEGF interact via the activation of PI3K/Akt signaling pathway, and changes in their expression are involved in the occurrence and development of GISTs. Thus, these factors may be suitable drug targets, because suppression of angiogenesis will decrease tumor blood supply and inhibit metastasis.

Finally, our study of GIST cells also showed that up-regulation of uPA activated MMP-2 and MMP-9, two proteins known to be involved in degradation of the extracellular matrix [26]. The expressions of uPA, MMP-2, and MMP-9 increased with the extent of mucosal invasion, metastasis, and tumor grade, but were independent of gender, age, and histological features. These findings suggest that TGF- $\beta_{1}$, uPA, MMP-2, and MMP-9 are also involved in the invasion and metastasis of GISTs.

The present research represents an initial step toward development of a more comprehensive program to identify genes that have a role in GIST and therefore have potential use in the diagnosis, prediction of outcome, and development of potential therapeutic targets.

Acknowledgements: Liaoning Department of Education (Project No.: 2009A726).

\section{References}

[1] LAURINI JA, CARTER JE. Gastrointestinal stromal tumors. Arch. Pathol. Lab. Med. 2010; 134: 134-141.

[2] NGUYEN SQ, DIVINO CM, WANG JL, DIKMAN SH. Laparoscopic management of gastrointestinal stromal tumors. Surg. Endosc. 2006; 20: 713-716. http: //dx.doi.org/10.1007/ s00464-005-0435-8

[3] AN JY, CHOI MG, NOH JH, SOHN TS, KANG WK, et al. Gastric GIST: A single institutional retrospective experience with surgical treatment for primary disease. EJSO. 2007; 33: 1030-1035. http: //dx.doi.org/10.1016/j.ejso.2007.02.009

[4] EDLING CE, HALLBERG B. c-Kit--a hematopoietic cell essential receptor tyrosine kinase. Int. J. Biochem. Cell Biol. 2007; 39: 1995-1998. http: //dx.doi.org/10.1016/ j.biocel.2006.12.005
[5] BAUER S, DUENSING A, DEMETRI GD, FLETCHER JA. KIT oncogenic signaling mechanisms in imatinab-resistant gastrointestinal stromal tumor: PI3-kinase/AKT is a cucial survival pathway. Oncogene. 2007; 26: 7560-7568. http: //dx.doi.org/10.1038/sj.onc. 1210558

[6] YUAN TL, CANTLEY LC. PI3K pathway alterations in cancer: variations on a theme. Oncogene. 2008; 27: 5497-5510. http: //dx.doi.org/10.1038/onc.2008.245

[7] CARNERO A, BLANCO-APARICIO C, RENNER O, LINK W, LEAL JF et al. The PTEN/PI3K/AKT Signalling Pathway in Cancer, Therapeutic Implications. Curr. Cancer Drug Targets 2008; 8: 187-198. http: //dx.doi.org/10.2174/ $\underline{156800908784293659}$

[8] CARRACEDO A, PANDOLFI PP. The PTEN-PI3K pathway: of feedbacks and cross-talks. Oncogene. 2008; 27: 5527-5541. http: //dx.doi.org/10.1038/onc.2008.247

[9] KESSENBROCK K, PLAKS V, WERB Z. Matrix metalloproteinases: regulators of the tumor microenvironment. Cell 2010; 141: 52-67. http: //dx.doi.org/10.1016/j.cell.2010.03.015

[10] CARNERO A, BLANCO-APARICIO C, RENNER O, LINK W, LEAL JF et al. The PTEN/PI3K/AKT signalling pathway in cancer, therapeutic implications. Curr. Cancer Drug Targets 2008; 8: 187-198. http: //dx.doi.org/10.2174/ $\underline{156800908784293659}$

[11] MORBIDELLI L, DONNINI S, ZICHE M. Role of nitric oxide in tumor angiogenesis. Cancer Treat Res. 2004; 117: 155-167. http: //dx.doi.org/10.1007/978-1-4419-8871-3 11

[12] BALUK P, HASHIZUME H, MCDONALD DM. Cellular abnormalities of blood vessels as targets in cancer. Curr. Opin. Genet. Dev. 2005; 15: 102-111. http: //dx.doi.org/10.1016/ j.gde.2004.12.005

[13] BALUK P, HASHIZUME H, MCDONALD DM. Cellular abnormalities of blood vessels as targets in cancer. Curr. Opin. Genet. Dev. 2005; 15: 102-111. http: //dx.doi.org/10.1016/ j.gde.2004.12.005

[14] FLETCHER CD, BERMAN JJ, CORLESS C, GORSTEIN F, LASOTA J et al. Diagnosis of gastrointestinal stromal tumors: a consensus approach. Int. J. Surg. Pathol. 2002; 10: 81-89. http: //dx.doi.org/10.1177/106689690201000201

[15] WEIDNER N. Tumor angiogenesis: review of current applications in tumor prognostication. Semin. Diagn. Pathol. 1993; 10: 302-313.

[16] CRISTINA R. ANTONESCU. Targeted therapies in gastrointestinal stromal tumors. Semin. Diagn. Pathol. 2008; 25: 295-303. http: //dx.doi.org/10.1053/j.semdp.2008.08.004

[17] YANG J, DU X, LAZAR AJ, POLLOCK R, HUNT K et al. Genetic aberrations of gastrointestinal stromal tumors. Cancer 2008; 113: 1532-1543. http: //dx.doi.org/10.1002/ $\underline{\text { cncr. } 23778}$

[18] WAITE KA, ENG C. Protean PTEN: form and function. Am. J. Hum. Genet. 2002; 70: 829-844. http: //dx.doi.org/10.1086/ $\underline{340026}$

[19] BEL AIBA RS, DIMOVA EY, GORLACH A, KIETZMANN T. The role of hypoxia inducible factor- 1 in cell metabolism--a possible target in cancer therapy. Expert Opin. Ther. Targets 2006; 10: 583-599. http: //dx.doi.org/10.1517/ $\underline{14728222.10 .4 .583}$ 
[20] RADISAVLJEVIC Z. AKT as locus of fragility in robust cancer system. J. Cell Biochem. 2008; 104: 2071-2077. http: //dx.doi. org/10.1002/jcb.21777

[21] BERTA M, BRAHIMI-HORN C, Pouyssegur J. Regulation of the Hypoxia-Inducible Factor-1alpha (HIF-1alpha): a breath of fresh air in hypoxia research. J. Soc. Biol. 2004; 198: 113-120.

[22] TAKAHASHI R, TANAKA S, KITADAI Y, SUMII M, YOSHIHARA $M$ et al. Expression of vascular endothelial growth factor and angiogenesis in gastrointestinal stromal tumor of the stomach. Oncology 2003; 64: 266-274. http: //dx.doi. org $/ 10.1159 / 000069316$

[23] LI L, WANG L, ZHANG W, TANG B, ZHANG J et al. Correlation of serum VEGF levels with clinical stage, therapy efficacy, tumor metastasis and patient survival in ovarian cancer. Anticancer. Res. 2004; 24: 1973-1979.

[24] SARTELET H, OLIGNY LL, VASSAL G. AKT pathway in neuroblastoma and its therapeutic mplication. Expert Rev. Anticancer. Ther. 2008; 8: 757-769. http://dx.doi.org/10.1586/ $\underline{14737140.8 .5 .757}$

[25] FITZPATRICK B, MEHIBEL M, COWEN RL, STRATFORD IJ. iNOS as a therapeutic target for treatment of human tumors. Nitric. Oxide. 2008; 19: 217-224. http: //dx.doi. org/10.1016/j.niox.2008.05.001

[26] JOHN A, TUSZYNSKI G. The role of matrix metalloproteinases in tumor angiogenesis and tumor metastasis. Pathol. Oncol. Res. 2001; 7: 14-23. http://dx.doi.org/10.1007/ $\underline{\text { BF03032599 }}$ 